\title{
DUST IN H II REGIONS
}

\author{
VAHE PETROSIAN
}

Institute for Plasma Research, Stanford University, Stanford, Calif., U.S.A.

\begin{abstract}
The observed far infrared flux of $\mathrm{H}$ II regions is interpreted as evidence for the presence of internal dust absorbing a fraction of Lyman continuum photons. The effect of this dust on the required number of ionizing photons and on the helium to hydrogen line intensity ratios are discussed and analytic formulae describing these effects are presented for simplified models.
\end{abstract}

\section{Introduction}

The purpose of this paper is to discuss the effects of the presence of dust in $\mathrm{H} \mathrm{II}$ regions and in other gaseous nebulae on the ionization structures of these nebulae. I consider a spherically symmetric nebula with a single ionizing star located at its center, and present here analytic expressions describing two effects. First I shall discuss how the presence of dust alters the required number of ionizing photons. This effect depends on only one parameter, namely the total absorption optical depth $\tau_{D}$ due to dust throughout the nebula averaged over all frequencies beyond the Lyman limit. If $n_{D}$ is the number density of dust and $r_{\mathrm{o}}$ is the observed radius of the nebula then

$$
\tau_{D}=\int_{0}^{r_{o}} n_{D} \bar{\sigma}_{D} \mathrm{~d} r
$$

and

$$
\bar{\sigma}_{D}(r)=\int_{v_{1}}^{\infty} \sigma_{D}(v) N(r, v) \mathrm{d} v / \int_{v_{1}}^{\infty} N(r, v) \mathrm{d} v,
$$

where $N(r, v)$ is the net flux of photons (per sec per frequency interval) flowing through a shell of radius $r$ and $v_{1}$ is the frequency of the Lyman limit.

The second effect I consider here is how the presence of dust alters the ratios of the Strömgren radii of other elements to that of hydrogen. In particular I shall consider the change in this ratio for helium which depends on the relative values of optical depths $\tau_{D}$ and $\tau_{D}^{\prime}$, the absorption optical depth for frequencies greater than $1.8 v_{1}\left(1.8 h v_{1}=\right.$ ionization potential of $\mathrm{He} \mathrm{I}$ ).

$$
\tau_{D}^{\prime}=\int_{0}^{r_{0}^{\prime}} n_{D} \bar{\sigma}_{D}^{\prime} \mathrm{d} r
$$

where $r_{o}^{\prime}$ is the helium Strömgren radius* and $\bar{\sigma}_{D}^{\prime}$ is the average dust absorption cross

* In this paper I assume that $r_{0}^{\prime} \leq r_{0}$ which is true for most galactic nebulae where the sources of ionization are stars of effective temperature $<6 \times 10^{4} \mathrm{~K}$ (Hummer and Seaton, 1964). I also neglect the presence of doubly ionized helium so that the results apply to regions of nebulae outside the He ill zones. 
section for frequencies above $1.8 v_{1}$, defined as in Equation (2) with $v_{1}$ replaced by $1.8 v_{1}$.

\section{Evidence for Dust in $H$ II Regions}

Before describing these effects let me discuss briefly the evidence for the presence of dust in $\mathrm{H}$ II regions. In the first place, if the ratio of gas to dust in the $\mathrm{H}$ il regions (typically for bright $\mathrm{H}$ II regions the hydrogen column density is few times $10^{21} \mathrm{~cm}^{-2}$ ) is the same as in the interstellar space, where there is on the average one magnitude visual extinction per $10^{21} \mathrm{~cm}^{-2} \mathrm{H}$-atoms in the line of sight, then we expect in $\mathrm{H} \mathrm{II}$ regions a visual optical depth (scattering and absorption) of greater than one and even greater optical depth for ultraviolet photons. Another evidence for dust in $\mathbf{H}$ II regions is provided by the work of O'Dell and his collaborators (O'Dell and Hubbard, 1965; O'Dell et al., 1966; Krishna Swamy and O'Dell, 1967) who discovered that the diffuse nebular emission is more than that expected from atomic processes. They attribute the excess to scattering of stellar light by dust grains in the nebula. These and other similar observations could be interpreted as evidence for dust which only scatters stellar radiation.

However, there are now observations indicating absorption by dust. Münch and Persson (1971), from high resolution observations of the differential reddening of the Balmer lines in Orion nebula, conclude that the dust is mixed with the gas and therefore it is absorption by dust which is responsible for the reddening. They also find that high gas density regions correspond to high dust density regions. Because of this evidence I shall assume in this discussion that the ratio of dust to gas densities is independent of position in the nebula.

Finally, an indirect evidence for absorbing dust in $\mathrm{H}$ II regions is their large far infrared fluxes, observed by Harper and Low (1971), by Hoffmann et al. (1971), and by Sofier et al. (1972). This flux is presumably due to dust. The question that arises now is what heats up the dust. One heating source is the Lyman alpha (L $\alpha)$ photons trapped in the $\mathrm{H}$ II regions. The expected infrared intensities due to $\mathrm{L} \alpha$ absorption are compared with the observed infrared intensities on Table I. The first column gives the infrared source numbers from Hoffman et al. (1971) and the second column gives their observed fluxes in the wavelength range 95 to $135 \mu$. Harper and Low (1971) have observed some of these sources in the wavelength range 45-750 $\mu$. Their reported fluxes are shown on the third column, which are on the average three times larger than the fluxes in the second column. Consequently, I have estimated the total far infrared fluxes of those sources not observed by Harper and Low by multiplying their 85 to $135 \mu$ fluxes by three. These numbers are shown in parenthesis. The fourth column gives the observed $5000 \mathrm{MHz}$ fluxes of these sources using the identifications of Hoffmann et al. (1971). Finally, the fifth column gives the expected L $\alpha$ fluxes calculated from the radio fluxes assuming that the nebulae are optically thick to $\mathrm{L} \alpha$ (case B). Comparison of column 5 and 3 shows that the expected infrared intensities due to absorption of $\mathrm{L} \alpha$ photons falls short of the observed intensities by factors ranging from 2 to 30 . Consequently, I conclude that a fraction of stellar photons are 
TABLE I

\begin{tabular}{|c|c|c|c|c|c|}
\hline \multicolumn{2}{|l|}{ Source } & \multirow{2}{*}{$\begin{array}{l}I_{\mathrm{IR}} \\
85-135 \mu \\
\operatorname{erg~cm} \mathrm{cm}^{-2} \mathrm{~s}^{-1}\end{array}$} & \multirow{2}{*}{$\begin{array}{l}I_{\mathrm{IR}} \\
\text { total } \\
\mathrm{erg} \mathrm{cm}^{-2} \mathrm{~s}^{-1}\end{array}$} & \multirow{2}{*}{ 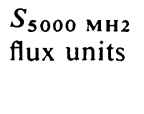 } & \multirow{2}{*}{$\begin{array}{l}I_{\mathrm{L} \alpha} \\
\mathrm{erg} \mathrm{cm}{ }^{-2} \mathrm{~s}^{-1}\end{array}$} \\
\hline $\begin{array}{l}\text { Hoffmann } \\
\text { et al. }\end{array}$ & $\begin{array}{l}\text { Other } \\
\text { name }\end{array}$ & & & & \\
\hline 6 & Orion A & $5.3 \times 10^{-6}$ & $18.6 \times 10^{-6}$ & 340 & $2.3 \times 10^{-6}$ \\
\hline 23 & & 0.92 & $(2.8)$ & 40 & 0.27 \\
\hline 26 & NGC6357 & 0.81 & (2.4) & 156 & 1.0 \\
\hline 27 & NGC6357 & 0.95 & $(2.8)$ & 215 & 1.4 \\
\hline 42 & & 1.14 & (3.4) & 15 & 0.10 \\
\hline 43 & & 1.00 & $(3.0)$ & 21 & 0.14 \\
\hline 46 & M8 & 0.51 & (1.5) & 100 & 0.66 \\
\hline 48 & W30 & 0.35 & (1.0) & 60 & 0.40 \\
\hline 49 & W31 & 0.71 & $(2.1)$ & 60 & 0.40 \\
\hline 50 & W33 & 0.63 & (1.9) & 40 & 0.26 \\
\hline 51 & & 0.62 & (1.9) & 30 & 0.20 \\
\hline 55 & M17 & 3.6 & 7.3 & 550 & 3.6 \\
\hline 56 & & 0.55 & (1.6) & 20 & 0.13 \\
\hline 57 & W43 & 1.03 & $(3.0)$ & 95 & 0.62 \\
\hline 58 & W49 & 1.14 & 3.1 & 50 & 0.33 \\
\hline 59 & W51a & 0.36 & 1.2 & 34 & 0.22 \\
\hline 60 & W51b & $1.95 \times 10^{-6}$ & $6.4 \times 10^{-6}$ & 100 & $0.66 \times 10^{-6}$ \\
\hline
\end{tabular}

absorbed by dust, so that $\tau_{D}>0$. The exact value of $\tau_{D}$ will depend on the fraction of infrared flux which is due to absorption by dust of Lyman continuum photons. This fraction depends on the spectrum of the central star and on the variation of albedo and total extinction of dust grains with frequency.

\section{Effects of Dust}

Let us now return to the calculation of the two effects described above. These calculations are similar to that described by Code (1973) and will not be discussed in detail here. In fact, in the present case the equations of the transfer of radiation are much simpler since I am interested only in photons which are absorbed completely in the $\mathrm{H}$ II region. As a result the scattering part of the cross section does not enter the calculations directly. The effect of scattering is to trap photons in the nebula and increase the effective optical depths (due to both gas and dust) to values larger than what one obtains from Equation (1). The effects of scattering can be neglected by replacing the line of sight absorption optical depths (Equation (1) with the effective absorption optical depths obtained from the integration along the photon paths.

(a) Effect of dust on the required number of ionizing photons: The net flux of ionizing photons crossing a spherical shell of radius $r$ is given by

$$
S(r)=\int_{v_{1}}^{\infty} N(r, v) \mathrm{d} v,
$$

with

$$
S\left(r_{o}\right)=0, \quad S(O) \equiv S^{*} .
$$


For a pure hydrogen nebula of density $n_{\mathrm{H}}(r)$, the variation of $S(r)$ with the radial coordinates $r$ is described by

$$
\frac{\mathrm{d} S(r)}{\mathrm{d} r}=-n_{D} \sigma_{D} S(r)-4 \pi \alpha^{(2)} \int_{0}^{r} x n_{\mathrm{H}} n_{e} r^{\prime 2} \mathrm{~d} r^{\prime},
$$

where $n_{e}$ is the electron density, $x$ is the fraction of hydrogen ionized and $\alpha^{(2)}$ is the recombination coefficient to excited levels of hydrogen.* The effect of dust is expressed by the first term on the right hand side of Equation (6). The rest of the terms in this equation are identical with the terms one obtains for a nondusty nebula (cf. Spitzer, 1968). In the absence of dust, Equation (6) gives the usual relationship between the Strömgren radius $r_{\mathrm{o}}$ and the number of ionizing photons emitted by the central star,

$$
S_{\mathrm{gas}}^{*}=4 \pi \alpha^{(2)} \int_{0}^{r_{0}} x n_{\mathrm{H}} n_{e} r^{2} \mathrm{~d} r .
$$

If we now introduce dust in this nebula, we will require a larger number of ionizing photons, since in addition to $S_{\text {gas }}^{*}$ photons absorbed by the gas, photons are absorbed also by dust. In order to see how the presence of dust changes the required value of ionizing photons let us look at the solution of Equation (6).

$$
S(r)=e^{-\tau}\left(S^{*}-\frac{4 \pi \alpha^{(2)}}{\bar{\sigma}_{D}} \int_{0}^{r} \frac{x n_{e} n_{\mathrm{H}}}{n_{D}} r^{\prime 2} e^{\tau^{\prime}} \mathrm{d} \tau^{\prime}\right),
$$

where $\mathrm{d} \tau=n_{D} \bar{\sigma}_{D} \mathrm{~d} r$. At the boundary of the $\mathrm{H}$ II region $S\left(r_{\mathrm{o}}\right)=0$, so that we require

$$
S^{*}=\frac{4 \pi \alpha^{(2)}}{\bar{\sigma}_{D}} \int_{0}^{\tau_{D}} \frac{x n_{e} n_{\mathrm{H}}}{n_{D}} r^{2} e^{\tau} \mathrm{d} \tau
$$

ionizing photons for a dusty nebula compared to $S_{\text {gas }}^{*}$ photons for a nebulae of the same size containing only gas. The presence of dust increases the required number of ionizing photons by a factor

$$
\frac{S^{*}}{S_{\text {gas }}^{*}}=\int_{0}^{\tau_{D}} g(r) e^{\tau} \mathrm{d} \tau / \int_{0}^{\tau_{D}} g(r) \mathrm{d} \tau, \quad g(r)=r^{2} n_{e} x n_{\mathrm{H}} / n_{D} .
$$

For a uniform nebula $\left(n_{\mathrm{H}}(r)=\right.$ const.) we find

$$
\frac{S^{*}}{S_{\mathrm{gas}}^{*}} \equiv f\left(\tau_{D}\right)=\frac{3 e^{\tau_{D}}}{\tau_{D}^{3}}\left[\tau_{D}^{2}-2 \tau_{D}+2\left(1-e^{-\tau_{D}}\right)\right] .
$$

This result was derived also independently by J. Silk and G. Field. This and some other effects of dust are discussed in a joint paper (Petrosian et al., 1972). The inverse of the quantity $f\left(\tau_{0}\right)$, which is the fraction of ionizing radiation absorbed by the gas,

* For a hydrogen nebula, the electron density $n_{e}=x n_{\mathrm{H}}$. For a nebula with both hydrogen and helium $n_{e}=x n_{\mathrm{H}}+y n_{\mathrm{He}}$, where $y$ is the fraction of He ionized, Equation (6) is still valid to a good approximation. This is because recombination coefficients for hydrogen and helium are approximately equal (Burgess and Seaton, 1960) and because each helium ionization is followed by a recombination which injects into the nebula approximately one photon capable of ionizing hydrogen (Robbins, 1970). 


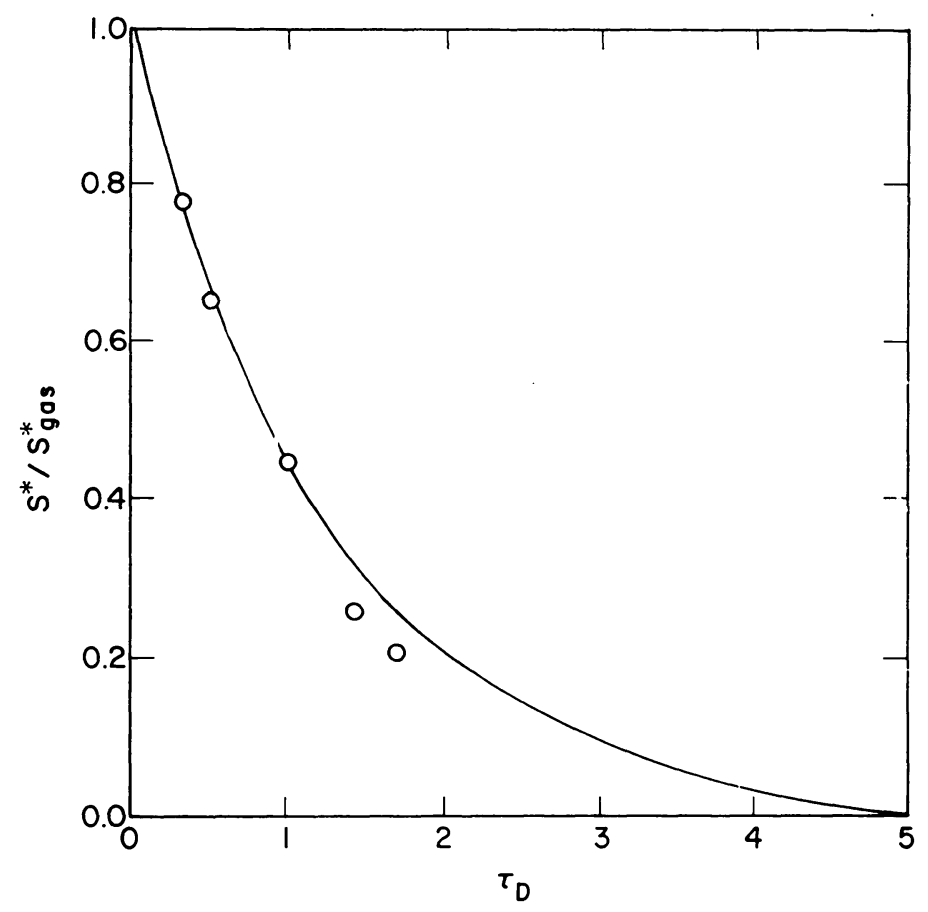

Fig. 1. Fraction of ionizing photons absorbed by gas vs the total effective absorption optical depth. The points are from the numerical results of Mathis (1971) which refer to the line of sight absorption - optical depths.

is plotted vs $\tau_{D}$ (the effective absorption optical depth of dust) on Figure 1. As can be seen, even for optical depths of about unity, by neglecting the effect of dust one underestimates the required ionizing flux by a factor of about 2 to 3 . For comparison with the expression (11) I have plotted on Figure 1 the points from numerical calculations of Mathis (1971). The discrepancies at large optical depths are due to scattering by dust which was included in Mathis' calculation. I have neglected the scattering by dust and have interpreted $\tau_{D}$ as the effective absorption optical depth (along the photon path) while points from Mathis' work refer to the absorption optical depth along the radius vector which is smaller than the effective optical depth when the albedo of dust is not equal to zero.

(b) Effect of dust on the ratio of helium to hydrogen Strömgren radii: In order to discuss this effect one must consider the fate of photons which can ionize only hydrogen (photons with $v_{1}<v<1.8 v_{1}$ ) and photons which can ionize both hydrogen and helium $\left(v>1.8 v_{1}\right)$ separately. I define the following quantities:

$$
\begin{gathered}
S_{1}(r)=\int_{v_{1}}^{1.8 v_{1}} N(v, r) \mathrm{d} v, \quad S_{2}(r)=\int_{1.8 v_{1}}^{\infty} N(v, r) \mathrm{d} v, \quad S_{2}\left(r_{0}^{\prime}\right)=0, \\
=S_{1}+S_{2}, \quad \gamma^{*}=S_{2}^{*} / S^{*}, \quad Y=n(\mathrm{He}) / n(\mathrm{H}), \quad R=\left(r_{0}^{\prime} / r_{0}\right)^{3} .(12)
\end{gathered}
$$

One can write differential equations similar to Equation (6) for $S_{1}$ and $S_{2}$ (and carry 
out the analysis leading to Equation (11) as before) except in the present case the integrands in the second terms on the right hand side are more compliacted functions of the radial coordinate. This complication, however, will not effect the changes in the ratio $R$ of the volumes of the Strömgren spheres caused by the presence of dust.* For a nondusty nebula this ratio, which I will call $R_{\text {gas }}$, depends primarily on the quantity $\gamma^{*} / Y$. It will also depend on the fraction of photons with $v>1.8 v_{1}$ that are absorbed by hydrogen. For small values of $\gamma^{*}\left(\gamma^{*} / Y<1\right)$ this fraction is negligible and $R_{\text {gas }} \approx \gamma^{*} / Y(1+Y)$. For $\gamma^{*} / Y \gg 1$ this ratio tends to unity. Hummer and Seaton (1964) have discussed this problem in detail. Their results can be summarized by

$$
R_{\mathrm{gas}}=\frac{\gamma^{*}}{Y} g\left(\bar{\gamma}, \gamma^{*}, Y\right), \quad g=\left\{\begin{array}{l}
\frac{1}{1+Y}, \frac{\gamma^{*}}{Y}<1 \\
\frac{Y}{\gamma^{*}}, \frac{\gamma^{*}}{Y} \gg 1,
\end{array}\right.
$$

where $\bar{\gamma}$ is the average value of $S_{2} / S$ throughout the nebula. This relation has been shown schematically by the dashed line on Figure 2 . As mentioned above, the exact shape of this curve is not essential for the derivation of the change in the value of $R$ due to dust.

For a dusty but uniform nebula an equation similar to Equation (13) still holds, but in this case the quantity on the right hand side is no longer equal to the ratio of the volumes, instead we have

$$
\int_{0}^{\tau^{\prime} D} \tau^{2} e^{\tau} \mathrm{d} \tau / \int_{0}^{\tau_{D}} \tau^{2} e^{\tau} \mathrm{d} \tau=\frac{\gamma^{*}}{Y} g,
$$

so that the ratio of the Strömgren volumes is

$$
R \equiv\left(\frac{\tau_{D}^{\prime}}{\tau_{D}}\right)^{3}=\frac{f\left(\tau_{D}\right)}{f\left(\tau_{D}^{\prime}\right)} R_{\mathrm{gas}},
$$

where $f$ is defined in Equation (9). In deriving Equation (15) I have assumed that $\bar{\sigma}_{D}=\bar{\sigma}_{D}^{\prime}$ and that the function $g$ is not changed by the presence of dust. For a given value of $R_{\text {gas }}$ and $\tau_{D}$, Equations (9) and (13), give the value of $\tau_{D}^{\prime}$ and the ratio $R$. The ratio $R$ is plotted on Figure ( 2 for various values of $\tau_{D}$. As is evident, the effect of dust is to bring this ratio closer to unity.

\section{Summary and Results}

Now let us see how the effects I have discussed here will change the helium to hydrogen

* For comparison with observation (optical or radio line intensity ratios) we need the ratio of emission measures

$$
R^{\prime}=\int_{0}^{r_{0}^{\prime}} n_{e} n_{\mathrm{He} \mathrm{II}} r^{2} \mathrm{~d} r / \int_{0}^{r_{0}} n_{e} n_{\mathrm{H} \mathrm{II}} r^{2} \mathrm{~d} r .
$$

For a uniform nebula $R^{\prime}=R Y$. 


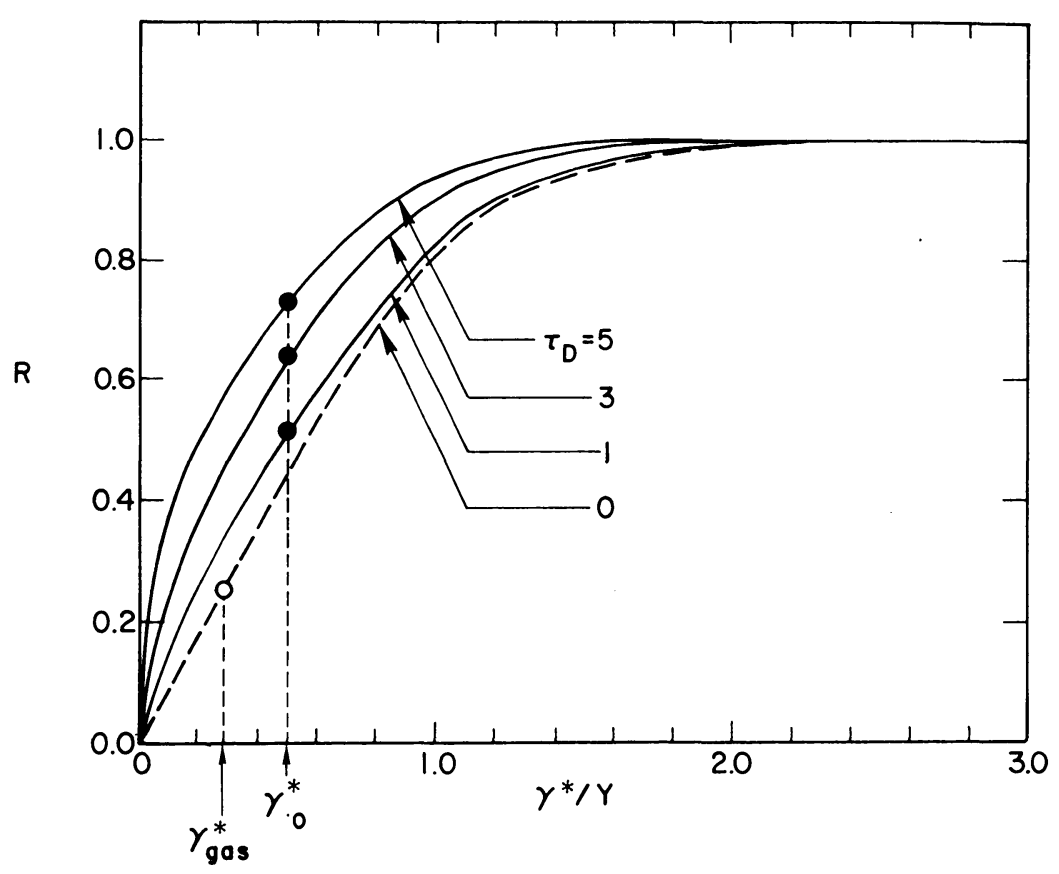

Fig. 2. The ratio of the values of Strömgren Spheres of helium to hydrogen versus the ratio of fraction of stellar ionizing photons capable of ionizing helium $\gamma^{*}$ to helium to hydrogen abundance ratio $Y$. For details see Section 4.

line intensity ratios. Let us consider an $\mathrm{H}$ il region of observed size $r_{\mathrm{o}}$, and an assumed abundance ratio $Y$. Neglecting the presence of dust one can calculate, from Equation (7), the required number of ionizing photons and find the spectral type of the ionizing star. From this and model atmospheric calculations (e.g. Auer and Mihalas, 1971) one finds the value of $\gamma_{\mathrm{gas}}^{*}$. The dashed line on Figure 2 then gives the expected value of the ratio $R_{\text {gas }}$ (the open circle on Figure 2 ) If there is absorbing dust of optical depth $\tau_{D}$ in the $\mathrm{H}$ II region we know that the required number of ionizing photons is increased by $f\left(\tau_{D}\right)$ (Equation (9)). This will require as an ionizing agent a new star (of larger effective temperature) which implies a new and in general a larger value for $\gamma^{*}$, say $\gamma_{0}^{*}$ (Figure 2). The correct value of the ratio $R$ is then obtained by reading on Figure 2 for $\gamma^{*}=\gamma_{0}^{*}$ the value of the ratio from the appropriate curve (filled circles). These two effects increase the ratio $R$ by a substantial factor, so that by neglecting dust, one underestimates the values of helium to hydrogen lines intensity ratios. This effect is more important for smaller values of $\gamma^{*} / Y$ and tends to zero for $\gamma^{*} / Y>1$, where both the dusty and nondusty models give a value near unity for the ratio $R$. It is believed that for bright $\mathrm{H}$ II regions the ratio $R$ is near unity. Whether this is due to a large value of $\gamma^{*} / Y$ or due to dust (or both) depends on various parameters which can vary from nebula to nebula.

In summary, I have interpreted the large far infrared flux observed from $\mathrm{H}$ II regions as emission by internal dust. My purpose here has been to point out the 
qualititive effects of this dust on the ionization structure of $\mathrm{H}$ II regions without attempting a detailed comparison with specific models. In particular I have attempted to indicate that most of these effects can be described by simple analytic formulae. I have not discussed here the possibility of evaluation of the ultraviolet properties of the dust from radio, infrared and optical observations. This is possible in principle but difficult in practice, because it is not clear what fraction of the heating of the dust is due to stellar photons below the Lyman limit and what fraction due to the ionizing photons considered here. In general, one can write for the difference between the observed infrared and the expected $\mathrm{L} \alpha$ luminosities the relation

$$
L(\mathrm{IR})-L(\mathrm{~L} \alpha)=S_{\mathrm{gas}}^{*}\left\langle h v_{\mathrm{Lc}}\right\rangle\left[f\left(\tau_{\mathrm{D}}\right)-1\right]+L^{*}\left(v<v_{1}\right)\left(1-e^{-\tau^{\prime \prime} D}\right),
$$

where $L^{*}\left(v<v_{1}\right)$ is the stellar luminosity below the Lyman limit, $\tau_{D}^{\prime \prime}$ is the dust absorption optical depth averaged over these frequencies and $\left\langle h v_{\mathrm{Lc}}\right\rangle$ is the average energy of stellar Lyman continuum photons. Since $S_{\text {gas }}^{*}=\left(\frac{3}{2}\right) L(\mathrm{~L} \alpha) / h v_{1}$ (case B), one can calculate the value of $\tau_{D}$ from Table I and Equation (11) if the last term on the right hand side of Equation (16) is negligible. This value of optical depth combined with scattering optical depths inferred from other observations can give an indication of the albedo of the dust. If, on the other hand, the last term in Equation (16) is not negligible, it is necessary to know the spectrum of the ionizing star in detail before one can proceed with the above calculation.

\section{Acknowledgements}

This work was supported by National Aeronautics and Space Administration Grant Number NGR 05-020-510.

\section{References}

Burgess, A. and Seaton, M. J.: 1960, Monthly Notices Roy. Astron. Soc. 121, 471.

Code, A.: 1973, this volume, p. 505.

Harper, D. A. and Low, F. J.: 1971, Astrophys. J. Letters 165, L9.

Hoffmann, W. F., Frederick, C. L., and Emery, R. J.: 1971, Astrophys. J. Letters 170, L89.

Hummer, D. G. and Seaton, M. J.: 1964, Monthly Notices Roy. Astron. Soc. $127,217$.

Krishna Swamy, K. S. and O'Dell, C. R.: 1967, Astrophys. J. 147, 529.

Mathis, J. S.: 1971, Astrophys. J. 167, 261.

Auer, L. H. and Mihalas, D.: 1971, Astrophys. J. Suppl. 24, 193.

Munch, G. and Persson, S. E.: 1971, Astrophys. J. 165, 241.

O'Dell, C. R. and Hubbard, W. B.: 1965, Astrophys. J. 142, 591.

O'Dell, C. R., Hubbard, W. B., and Peimbert, M.: 1966, Astrophys. J. 143, 743.

Petrosian, V., Silk, J., and Field, G. B.: 1972, Astrophys. J. Letters 177, L69.

Robbins, R. R.: 1970, Astrophys. J. 160, 519.

Soifer, B. T., Pipher, J. D., and Houck, J. R.: 1972, paper presented at the IAU Symp. 52 on 'Interstellar Dust and Related Topics', not published.

Spitzer, L., Jr.: 1968, in Diffuse Matter in Space, Interscience Publishers, Division of J. Wiley and Sons, New York, p. 115. 